\title{
Rapid Detection of Cercospora beticola in Sugar Beet and Mutations Associated with Fungicide Resistance Using LAMP or Probe-Based qPCR
}

\author{
Subidhya Shrestha, ${ }^{1,2}$ Jonathan Neubauer, ${ }^{2}$ Rebecca Spanner, ${ }^{1,2}$ Mari Natwick, ${ }^{2}$ Joshua Rios, ${ }^{2}$ Nicholas Metz, ${ }^{1,2}$ Gary A. Secor, ${ }^{1}$ \\ and Melvin D. Bolton ${ }^{1,2, \dagger}$ \\ ${ }^{1}$ Department of Plant Pathology, North Dakota State University, Fargo, ND 58108, U.S.A. \\ ${ }^{2}$ United States Department of Agriculture-Agricultural Research Service, Northern Crop Science Laboratory, Fargo, ND 58102, \\ U.S.A.
}

\begin{abstract}
Cercospora leaf spot (CLS), caused by the fungal pathogen Cercospora beticola, is the most destructive disease of sugar beet worldwide. Although growing CLS-tolerant varieties is helpful, disease management currently requires timely application of fungicides. However, overreliance on fungicides has led to the emergence of fungicide resistance in many $C$. beticola populations, resulting in multiple epidemics in recent years. Therefore, this study focused on developing a fungicide resistance detection "toolbox" for early detection of $C$. beticola in sugar beet leaves and mutations associated with different fungicides in the pathogen population. A loop-mediated isothermal amplification (LAMP) method was developed for rapid detection of $C$. beticola in infected sugar beet leaves. The LAMP primers specific to $C$. beticola (Cb-LAMP) assay was able to detect C. beticola in inoculated sugar beet leaves as early as 1 day postinoculation. A quinone outside inhibitor (QoI)-LAMP assay was also
\end{abstract}

Abstract developed to detect the G143A mutation in cytochrome $b$ associated with QoI resistance in C. beticola. The assay detected the mutation in C. beticola both in vitro and in planta with $100 \%$ accuracy. We also developed a probe-based quantitative PCR (qPCR) assay for detecting an E198A mutation in $\beta$-tubulin associated with benzimidazole resistance and a probe-based qPCR assay for detection of mutations in cytochrome P450-dependent sterol 14 $\alpha$-demethylase (Cyp51) associated with resistance to sterol demethylation inhibitor fungicides. The primers and probes used in the assay were highly efficient and precise in differentiating the corresponding fungicide-resistant mutants from sensitive wild-type isolates.

Keywords: benzimidazole, Cercospora beticola, Cercospora leaf spot (CLS), DMI, fungicide, G143A, loop-mediated isothermal amplification (LAMP), probe-based qPCR, sugar beet
Cercospora leaf spot (CLS) of sugar beet (Beta vulgaris L.), caused by Cercospora beticola Sacc., is the most destructive foliar disease of sugar beet in the world. CLS-infested sugar beet suffers a significant reduction in root yield and extractable sucrose from the loss of photosynthetic capacity and diversion of photosynthate for foliage regeneration (Franc 2010). The disease causes hundreds of millions of dollars in losses from yield reduction, quality deterioration, and higher processing and disease management costs (e.g., fungicide applications) annually (Secor et al. 2010; Steddom et al. 2005). Outbreaks of CLS are more frequent in regions with a warm and humid climate during the sugar beet growing season. CLS is a polycyclic disease with an ability to rapidly repeat the disease cycle during extended warm and humid weather. The fungus can produce conidia as early as 7 days from necrotic tissues that serve as a source of inoculum to start a new disease cycle (Bolton et al. 2012b; Franc 2010). This disease can be partially managed

Current address of N. Metz: Soil \& Crop Sciences, Colorado State University, Fort Collins, CO 80523, U.S.A.

${ }^{\dagger}$ Corresponding author: M. D. Bolton; melvin.bolton@usda.gov

Funding: This project was supported by United States Department of Agriculture-Agricultural Research Service Current Research Information System project number 3160-21000-044 and grants from the Sugarbeet Research and Education Board of Minnesota and North Dakota and the Beet Sugar Development Foundation.

*The $\boldsymbol{e}$-Xtra logo stands for "electronic extra" and indicates that six supplementary figures are published online.

The author(s) declare no conflict of interest.

Accepted for publication 28 January 2020.

This article is in the public domain and not copyrightable. It may be freely reprinted with customary crediting of the source. The American Phytopathological Society, 2020. by crop rotation and growing CLS-tolerant cultivars (Miller et al. 1994) but timely fungicide applications are still required in most sugar-beet-growing areas (Secor et al. 2010). However, excessive and repeated use of fungicides have imposed selection pressure on $C$. beticola populations, thus resulting in widespread occurrence of fungicide-resistant strains (Birla et al. 2012; Bolton et al. 2012a, 2013c, 2016).

The timing of fungicide application is critical and heavily dependent on risk predictions from disease forecasting models (Kaiser et al. 2010; Khan et al. 2007; Pethybridge et al. in press; Windels et al. 1998). However, disease forecasting models based on spore trapping or weather have had limited success in their predictive capabilities. This is likely because weather forecasting is not always dependable, and it is very difficult for disease prediction models to take into consideration the many variables that influence disease such as the levels of host resistance being used locally or how much the crop has canopied. Other prediction models utilize the previous day's temperature and relative humidity to predict the risk of CLS (Pitblado 2002). Consequently, the development of molecular tools that are sufficiently sensitive to identify the pathogen in asymptomatic leaves would confirm undoubtedly that the pathogen is present, which can then be followed up with molecular assays to identify mutations associated with fungicide resistance that will aid in fungicide application decisions.

C. beticola is considered a "high-risk" pathogen for developing fungicide resistance because of its high genetic variability, prolific sporulation, and polycyclic nature (Bolton et al. 2012a,b, 2013a; Secor et al. 2010). It is often observed that the efficacy of an extensively used fungicide is short lived. Fungicide resistance mechanisms are recurrently associated with nonlethal amino acid substitutions in pathogen genes that encode fungicide targets (Bolton et al. 2012a, 2013a; Davidson et al. 2006; Obuya et al. 2015; Secor et al. 2010; Trkulja et al. 2017). For example, resistance to the benzimidazole fungicide (Fungicide Resistance Action Committee [FRAC] group 1) thiophanate methyl caused a major CLS epidemic in North Dakota and Minnesota in 1981. Molecular analyses has shown that resistance to benzimidazole is associated with a glutamic acid to alanine 
substitution at position 198 (E198A) in C. beticola $\beta$-tubulin (Bolton et al. 2013b). Another important class of fungicides for CLS management are the quinone outside inhibitor (QoI) fungicides (FRAC group 11). QoIs inhibit mitochondrial respiration by binding to the quinol oxidation site of the cytochrome $b_{1}$ enzyme complex (complex III), thus disrupting the production of ATP, which causes an energy deficit in sensitive fungi (Gisi et al. 2002). The core of the cytochrome $b_{1}$ enzyme is encoded by the mitochondrial gene cytochrome $b(C y t b)$. A mutation conferring a glycine to alanine exchange at amino acid position 143 (G143A) in Cytb was found to be associated with QoI resistance in C. beticola (Birla et al. 2012; Bolton et al. 2013a,c).

Some fungal pathogens may rely on multiple mechanisms for fungicide resistance. For example, sterol demethylation inhibitor (DMI) fungicides (FRAC group 3) act by inhibiting cytochrome P450dependent sterol $14 \alpha$-demethylase (Cyp51), an enzyme required for ergosterol biosynthesis in fungi (Cools et al. 2013). However, repeated use of DMIs has resulted in resistance in many fungal pathogens, including C. beticola (Bolton et al. 2012a,b). Resistance to DMIs has been associated with amino acid substitutions in CYP51 (Cools et al. 2010; Trkulja et al. 2017), overexpression of Cyp51 (Bolton et al. 2016; Cools et al. 2012; Ma and Tredway 2013), and enhanced active efflux of fungicide from the cell due to increased expression of genes encoding a membrane ATP-binding cassette or major facilitator superfamily transporters (Kretschmer et al. 2009; Omrane et al. 2017; Zwiers et al. 2002). We reported previously that induced overexpression of CbCyp51 was associated with DMI resistance in C. beticola (Bolton et al. 2012a, 2016). More recently, we utilized a genome-wide association study to identify other mutations associated with resistance, including a leucine to phenylalanine exchange at position 144 (L144F) in CYP51 (R. Spanner and M. D. Bolton, unpublished).

As mentioned above, the identification of pathogens in asymptomatic leaves is critical to help guide fungicide application decisions for disease management. Techniques to isolate the pathogen from infected leaves are laborious and take too long for in-season fungicide recommendations. However, a relatively new technique called loop-mediated isothermal amplification (LAMP) is faster and more efficient, and can more accurately detect pathogens or mutations associated with fungicide resistance compared with conventional methods (Nagamine et al. 2002; Ordóñez et al. 2019; Vielba-Fernández et al. 2019). LAMP amplifies DNA at a single temperature in a short time period (approximately $45 \mathrm{~min}$ ) utilizing a set of four to six primers and a DNA polymerase with strand displacement activity (Goto et al. 2009). Due to the simplicity and robustness of the technique, LAMP can potentially be used for in-field diagnostics (Kogovšek et al. 2015).

This study focused on the development of a fungicide resistance detection "toolbox" containing: (i) LAMP- and quantitative PCR (qPCR)-based diagnostic methods to detect $C$. beticola in infected sugar beet leaves; (ii) LAMP-based detection of QoIresistant isolates in infected sugar beet plants; and probe-based qPCR methods for the detection of (iii) DMI-resistant and (iv) benzimidazole-resistant isolates in infected sugar beet plants. Taken together, this toolbox can be utilized to monitor fungicide resistance to three major chemistries used for CLS disease management.

\section{Materials and Methods}

Fungal isolates and DNA extraction. In total, 20 different $C$. beticola isolates collected throughout the Red River Valley of North Dakota and Minnesota in 2016 exhibiting varying fungicide sensitivities were used in the study (Table 1). Additionally, we utilized isolates of Alternaria alternata and Phoma betae as controls (Table 1). For DNA extraction, single spore-derived isolates were grown in clarified V8-medium plates, consisting of $10 \%$ (vol/vol) clarified V8 juice (Campbell's Soup Co.), $0.5 \%$ (wt/vol) $\mathrm{CaCO}_{3}$, and $1.5 \%$ (wt/vol) agar (MoorAgar, Inc.), for a week at $18^{\circ} \mathrm{C}$. For each isolate, four agar plugs $(5 \mathrm{~mm}$ each) were removed from the leading edge of colony growth and were transferred to $50 \mathrm{ml}$ of half-strength potato dextrose broth (BD Difco) and grown for 7 days at $18^{\circ} \mathrm{C}$. Mycelia were harvested by filtering through two layers of Miracloth (Calbiochem), flash frozen in liquid nitrogen, and ground using a Retsch Mixer Mill (Retsch) at a frequency of 30 oscillations/s for $1 \mathrm{~min}$. Ground mycelia were subsequently stored at $-80^{\circ} \mathrm{C}$ until use for DNA isolations. DNA was isolated using $100 \mathrm{mg}$ of ground mycelium from each isolate or $100 \mathrm{mg}$ of sugar beet leaf tissue with the DNeasy Plant Mini kit (Qiagen) according to the manufacturer's instructions. DNA concentrations were quantified using the Qubit BR DNA kit in the Qubit 3.0 fluorometer (Invitrogen). All DNA samples were brought to a concentration of $40 \mathrm{ng} \mu \mathrm{l}^{-1}$ using AE buffer (elution buffer) from the DNeasy Plant Mini kit.

Development of LAMP methodology for $C$. beticola detection. The fungal pathogens A. alternata, P. betae, and Ramularia beticola can infect sugar beet and can be misidentified as $C$. beticola in the absence of proper diagnostic tools. Therefore, to develop LAMP primers specific to $C$. beticola (Cb-LAMP), ribosomal DNA (rDNA) sequences and Cyp51 were chosen as candidate loci for diagnostic purposes. The rDNA sequences of $C$. beticola (accession numbers MF681167.1, MF681115.1, and AY840527.2), A. alternata (accession numbers AF455406.1 and AF392982.1), P. betae (accession numbers MK249659.1, KX239986.1, and KM249076.1), R. beticola (accession numbers KX287337.1, MH855080.1, and KX287344.1), and sugar beet (accession numbers AF161096.1, HE578879.1, and FJ669720.1) were obtained from NCBI GenBank. The Cyp51 gene sequence was obtained for $C$. beticola (accession numbers HM778021.1 and JN399035.1), A. alternata (accession numbers XM_018526615.1 and XM_018531400.1), and sugar beet (NCBI accession numbers XM_010693692.2, XM_010693994.2, and XM_010695831.2). Sequences were aligned using the MUSCLE Alignment option within

Table 1. Isolates used in this study ${ }^{\mathrm{a}}$

\begin{tabular}{|c|c|c|c|c|c|}
\hline \multirow{2}{*}{$\begin{array}{l}\text { Isolate } \\
\text { ID }\end{array}$} & \multirow[b]{2}{*}{ Species } & \multirow{2}{*}{$\frac{\text { QoI }}{\text { G143A }^{\mathbf{b}}}$} & \multirow{2}{*}{$\frac{\text { Benzimidazole }}{\text { E198A }^{\mathbf{c}}}$} & \multicolumn{2}{|c|}{ DMI } \\
\hline & & & & $\mathrm{L}^{144 F^{\mathrm{d}, \mathrm{e}}}$ & E170 $^{\mathrm{d}}$ \\
\hline 22 & $\begin{array}{c}\text { Cercospora } \\
\text { beticola }\end{array}$ & - & - & $+(\mathrm{T})$ & - \\
\hline 24 & C. beticola & + & + & $+(\mathrm{T})$ & - \\
\hline 44 & C. beticola & - & + & $+(\mathrm{C})$ & + \\
\hline 49 & C. beticola & - & - & - & + \\
\hline 57 & C. beticola & - & + & - & + \\
\hline 58 & C. beticola & - & + & - & + \\
\hline 61 & C. beticola & - & + & - & + \\
\hline 62 & C. beticola & + & - & - & - \\
\hline 63 & C. beticola & + & - & $+(\mathrm{T})$ & - \\
\hline 64 & C. beticola & + & + & - & - \\
\hline 65 & C. beticola & - & + & - & + \\
\hline 67 & C. beticola & + & - & $+(\mathrm{C})$ & - \\
\hline 68 & C. beticola & - & - & - & - \\
\hline 81 & C. beticola & - & - & - & + \\
\hline 82 & C. beticola & - & + & - & + \\
\hline 83 & C. beticola & - & + & - & + \\
\hline 86 & C. beticola & + & + & - & + \\
\hline 90 & C. beticola & + & + & - & - \\
\hline 91 & C. beticola & + & + & - & - \\
\hline $1-90$ & C. beticola & + & + & - & - \\
\hline $5 \mathrm{~A}$ & $\begin{array}{c}\text { Alternaria } \\
\text { alternata }\end{array}$ & NA & NA & NA & NA \\
\hline $\mathrm{Pb}-4$ & Phoma betae & NA & NA & NA & NA \\
\hline
\end{tabular}

${ }^{\mathrm{a}} \mathrm{QoI}=$ quinone outside inhibitor, $\mathrm{DMI}=$ sterol demethylation inhibitor, + indicates the Cercospora beticola isolate resistant to the corresponding fungicide chemistry, - indicates the $C$. beticola isolate sensitive to the corresponding fungicide chemistry, and NA = information pertaining to fungicide resistance in these isolates is not available.

b Mutation in C. beticola cytochrome b encoding a G143A substitution.

${ }^{c}$ Mutation in C. beticola $\beta$-tubulin encoding a E198A substitution.

${ }^{\mathrm{d}}$ Mutation in C. beticola Cyp51 encoding a L144F or E170 substitution.

e The $C$. beticola $\mathrm{L} 144 \mathrm{~F}$ substitution has two versions; (T) refers to the T-type and $(\mathrm{C})$ refers to the $\mathrm{C}$-type. 
Geneious Prime software package (v. 2019.0.04) to identify unique regions for primer development. LAMP primers were designed, in part, using PrimerExplorer (v5) (http://primerexplorer.jp/e/; Eiken Chemical Co., Ltd.) using default parameters. Two sets of $C$. beticola-specific LAMP primers were designed on the rDNA sequence (Cb-LAMP-1 and Cb-LAMP-2) and another two sets of LAMP primers were designed on Cyp51. Cb-LAMP assays were conducted using the WarmStart Colorimetric LAMP 2X Master Mix (DNA and RNA) kit (New England Biolabs) with $0.2 \mu \mathrm{M}$ each outer primer (F3 and B3) and 1.6 $\mu \mathrm{M}$ each internal primer (forward internal primer [FIP] and backward internal primer [BIP]) in a $25-\mu l$ final reaction volume. Preliminary tests were conducted to identify the most efficient and accurate primer set by testing on $C$. beticola isolates 49 and 57, one isolate of A. alternata, one isolate of $P$. betae (Table 1), and sugar beet DNA, which showed that the $\mathrm{Cb}$ LAMP-2 primer set $(2275,2276,2277$, and 2278) (Table 2) designed on $C$. beticola rDNA sequence was the best (see Results). Consequently, all further optimization strategies were focused on the Cb-LAMP-2 primer set (Table 2). The LAMP procedure was optimized using the Cb-LAMP-2 primer set and C. beticola DNA. Parameters that were assessed included incubation temperature $\left(61\right.$ to $69^{\circ} \mathrm{C}$ ), incubation time (20 to $\left.90 \mathrm{~min}\right)$, and concentration of template DNA (100 $\mathrm{ng}$ to $100 \mathrm{fg}$ ). All reactions were terminated with a deactivation step by incubating at $80^{\circ} \mathrm{C}$ for $5 \mathrm{~min}$. Positive reactions are represented by a change of pink to yellow color while negative reactions stay pink. LAMP amplification products were further visualized for the presence of typical ladder-like bands using $2.0 \%(\mathrm{wt} / \mathrm{vol})$ agarose gel electrophoresis.

To validate LAMP results, a qPCR methodology was developed using a subset of the primers used for the Cb-LAMP reaction. Specifically, the optimized procedure used for $C$. beticola detection in this study utilized the GoTaq qPCR Master Mix kit (Promega Corp.) in a $20-\mu l$ volume containing $1 \times$ Master Mix and $200 \mathrm{nM}$ primers 2275 and 2431 targeting rDNA of $C$. beticola or primers 2362 and 2345 targeting sugar beet rDNA (Table 2). qPCR conditions were $95^{\circ} \mathrm{C}$ for $10 \mathrm{~min}$, followed by 40 cycles of $95^{\circ} \mathrm{C}$ for $15 \mathrm{~s}$ and $63^{\circ} \mathrm{C}$ for $30 \mathrm{~s}$. All qPCR assays were performed in a Bio-Rad CFX96 machine (Bio-Rad Laboratories) and analyzed using Bio-Rad CFX Manager (Bio-Rad Laboratories). To validate the qPCR parameters and determine the detection limit for the Cb-qPCR assay, DNA from $C$. beticola isolate 57 (Table 1) was diluted in a 10-fold dilution series ranging from $100 \mathrm{ng}$ to $100 \mathrm{fg}$ and used to calculate efficiencies (Broeders et al. 2014; Bustin et al. 2009). The relative fungal biomass of $C$. beticola in infected sugar beet leaf samples was calculated as described by Pfaffl (2001). This experiment was conducted three times.

Development of LAMP methodology for QoI resistance detection in $\boldsymbol{C}$. beticola. We were interested in developing a LAMP assay

Table 2. Primers and probes used in this study

\begin{tabular}{|c|c|c|c|}
\hline Primer & Role $^{\mathbf{a}}$ & $\operatorname{Amp}(\mathbf{b p})^{\mathbf{b}}$ & Primer/probe sequence $\left(5^{\prime} \text { to } 3^{\prime}\right)^{c}$ \\
\hline \multicolumn{4}{|c|}{ Cb-LAMP-2 } \\
\hline 2275 & $\mathrm{~F} 3$ & $\ldots$ & TGAGGGCCTTCGGGCT \\
\hline 2276 & B3 & $\ldots$ & TCGCTGCGTTCTTCATCG \\
\hline 2277 & FIP & $\ldots$ & GCTCGCCGTCGAAACGGCATCCAACCCTTTGTGAACACA \\
\hline 2278 & BIP & $\ldots$ & ACTGCATCTTTGCGTCGGAGTATGCCAGAACCAAGAGATCC \\
\hline \multicolumn{4}{|c|}{$\mathrm{Cb}-\mathrm{qPCR} \mathrm{e}^{\mathrm{e}}$} \\
\hline 2275 & Cb-qPCR-For & 174 & TGAGGGCCTTCGGGCT \\
\hline 2431 & Cb-qPCR-Rev & 174 & ACTCCGACGCAAAGATGCAGT \\
\hline 2362 & Sb-qPCR-For & 165 & TAGATAAAAGGTCAACGCGGGCT \\
\hline 2345 & Sb-qPCR-Rev & 165 & AATTCTCCGTCACCCGTCACC \\
\hline \multicolumn{4}{|c|}{ QoI-LAMPf } \\
\hline 2112 & F3 & $\ldots$ & TCTTGGGTTATGTTTTACCTTAC \\
\hline 2113 & B3 & $\ldots$ & CCTGCACTATCATGTAAAGCA \\
\hline 2114 & FIP mut & $\ldots$ & CCTACTCATGGTATTGCACTCATTAGGACAAATGTCTTTATGACC \\
\hline 2118 & BIP & $\ldots$ & GAGGAGGTTTTTCTGTTAATAACGCGCTAATGCAGCTAATACGĀAAG \\
\hline \multicolumn{4}{|r|}{ 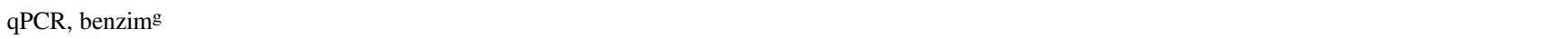 } \\
\hline 912 & Cb- $\beta$ tub-qPCR-For & 125 & CACTCTGTCCGTTCACCA \\
\hline 913 & Cb-ßtub-qPCR-Rev & 125 & GGTTCAAGTCGCCGTAAG \\
\hline 1933 & E198A mut-P & $\ldots$ & HEX-TCGTCGAGAACTCCGACG[+ㄷ]GA-BHQ \\
\hline 1935 & E198A WT-P & $\ldots$ & 6FAM-AACTCCGACG[+A]GACCTTCTG-BHQ- \\
\hline \multicolumn{4}{|c|}{ qPCR, DMI ${ }^{\mathrm{h}}$} \\
\hline 2288 & Cb-Cyp51-qPCR-For & 212 & AACTGCGCGATGTCAATG \\
\hline 2250 & Cb-Cyp51-qPCR-Rev & 212 & TCGCTTATGTGGGTTGTTCTT \\
\hline 2257 & E170 WT-P & $\ldots$ & 6FAM-AATTGGCGCGTCETCTTCG-BHQ-1 \\
\hline 2258 & E170 mut-P & $\ldots$ & HEX-AATTGGCGCGTTT̄CTTCG-BHQ-1 \\
\hline 2373 & L144F WT-P & $\ldots$ & HEX-CCCCAACTC/ZEN̄/CAAATTGATGG-IBFQ \\
\hline 2284 & L144F-C-P & $\ldots$ & 6FAM-CCCCAACTC/ZEN/CAAATTㅡATGG-IBFQ \\
\hline 2445 & L144F-T-P & $\ldots$ & TexRd-X-CCCCAACTCCAAATTTATTGGAG-IBRQ \\
\hline
\end{tabular}

${ }^{a}$ F3 = forward outer primers, $\mathrm{B} 3=$ backward outer primers, $\mathrm{FIP}=$ forward internal primers, $\mathrm{BIP}=$ backward internal primers; mut $=$ primer or probe with mutation site, $\mathrm{WT}=$ wild type, $\mathrm{For}=$ forward primers, $\mathrm{Rev}=$ reverse primers, and $\mathrm{P}=$ probe

b PCR amplicon size in base pairs, where appropriate.

c 6 FAM = 6-carboxyfluorescein, HEX = hexachloro-6-carboxyfluorescein, TexRd = Texas Red, BHQ-1 = black hole quencher-1, ZEN = ZEN internal quencher, $\mathrm{IBFQ}=$ Iowa Black FQ, IBRQ = Iowa Black RQ, underlined letters = nucleotide in mutation site, bold letter = mismatched nucleotide, and letters in brackets = locked nucleic acid. All primers were synthesized by Invitrogen and all probes were synthesized by Integrated DNA Technologies unless explained otherwise.

${ }^{\mathrm{d}} \mathrm{Cb}$-LAMP refers to detection of Cercospora beticola using loop-mediated isothermal amplification (LAMP) targeting ribosomal DNA (rDNA) of $C$. beticola.

${ }^{\mathrm{e}} \mathrm{Cb}-\mathrm{qPCR}$ refers to detection of $C$. beticola targeting rDNA using real-time quantitative PCR method. Cb-qPCR-Rev represents the reverse complement of B1c included in BIP primer of Cb-LAMP primer and Sb-rDNA indicates the primers targeting sugar beet rDNA.

${ }^{\mathrm{f}}$ QoI-LAMP refers to detection of resistance to quinone outside inhibitor (QoI) fungicides targeting G143A mutation in cytochrome b $(C y t b)$ of $C$. beticola using LAMP.

g Probe-based qPCR for the detection of E198A mutation in $\beta$-tubulin associated with benzimidazole resistance in C. beticola. Probes used in the assay were synthesized by Sigma-Aldrich.

${ }^{\mathrm{h}}$ Probe-based qPCR for the detection of sterol demethylation inhibitor (DMI) resistance. Detection of L144F and silent mutation E170 in cytochrome P450dependent sterol $14 \alpha$-demethylase (Cyp51) associated with resistance of $C$. beticola to DMI fungicide. 
to quickly detect the G143A mutation associated with QoI resistance in C. beticola. LAMP primers were designed, in part, using PrimerExplorer. The assay was developed so that QoI-resistant strains would allow for the amplification of the Cytb target sequence, thereby turning the LAMP reaction yellow. Three FIP primers were tested; each FIP primer included the cytosine nucleotide associated with the G143A mutation at the $3^{\prime}$ end of the primer. Additionally, a mismatched base was included at the penultimate position of each primer to more effectively discriminate QoI-resistant isolates from sensitive isolates (Supplementary Fig. S1). To test these primers in the QoI-LAMP assay, $0.17 \mu \mathrm{M}$ each outer primer F3 and B3 (Table 2), $1.33 \mu \mathrm{M}$ each internal primer FIP and BIP (Table 2), $1 \times$ Colorimetric LAMP Master Mix (NEB), and $10 \mathrm{ng}$ of template DNA was used. The QoI-LAMP procedure was optimized using $C$. beticola DNA from isolates 65 and 67 (Table 1). Parameters that were assessed included incubation temperature $\left(61\right.$ to $\left.69^{\circ} \mathrm{C}\right)$, incubation time (15 to $60 \mathrm{~min}$ ), and concentration of template DNA (100 ng to $100 \mathrm{fg}$ ). All reactions were terminated with a deactivation step by incubating at $80^{\circ} \mathrm{C}$ for $5 \mathrm{~min}$. Positive reactions (indicating QoI resistance) were represented by a change of pink to yellow color while negative reactions stayed pink. LAMP amplification products were further visualized for the presence of typical ladder-like bands using $2.0 \%(\mathrm{wt} / \mathrm{vol})$ agarose gel electrophoresis. The optimized procedure was incubation at $65^{\circ} \mathrm{C}$ for $45 \mathrm{~min}$ followed by deactivation at $80^{\circ} \mathrm{C}$ for 5 min. To validate QoI-LAMP results, the probe-based qPCR methodology developed by Bolton et al. (2013a) was utilized. To determine the detection limit for the QoI-LAMP assay, DNA from isolates 65 and 67 (Table 1) were diluted in a 10-fold dilution series ranging from $100 \mathrm{ng}$ to $100 \mathrm{fg}$. The LAMP reaction was incubated at $65^{\circ} \mathrm{C}$ for $60 \mathrm{~min}$ followed by deactivation at $80^{\circ} \mathrm{C}$ for $5 \mathrm{~min}$. LAMP reaction products were assessed for color change and visualized on a $2 \%(\mathrm{wt} / \mathrm{vol})$ agarose gel. This experiment was conducted three times.

Development of probe-based qPCR methodology for benzimidazole resistance detection in $\boldsymbol{C}$. beticola. To differentiate strains based on the E198A mutation, a set of dual-labeled probes was developed. For optimization, pairwise comparisons of primer $(100,200$, and $300 \mathrm{nM})$, probe $(50,100$, and $200 \mathrm{~nm}), \mathrm{MgCl}_{2}$ concentrations $(3.0,4.0$, and $5.0 \mathrm{mM})$, and annealing temperature $(55$ to $65^{\circ} \mathrm{C}$ ) were assessed. The optimized procedure used for screening isolates in this study utilized the GoTaq G2 Flexi DNA Polymerase kit in a $25-\mu \mathrm{l}$ volume containing $1 \times$ buffer, $4.0 \mathrm{mM} \mathrm{MgCl}_{2}, 0.3 \mathrm{mM}$ dNTPs, $100 \mathrm{nM}$ each primer (912 and 913) (Table 2), $50 \mathrm{nM}$ probe (1933 and 1935) (Table 2), $1.25 \mathrm{U}$ of GoTaq, and $10 \mathrm{ng}$ of genomic DNA. Probes 1933 and 1935 contained a locked nucleic acid on the mutation site and were supplied by Sigma-Aldrich. qPCR conditions were $95^{\circ} \mathrm{C}$ for $10 \mathrm{~min}$, followed by 40 cycles of $95^{\circ} \mathrm{C}$ for $15 \mathrm{~s}$ and $63^{\circ} \mathrm{C}$ for $45 \mathrm{~s}$. All qPCR assays were performed in a Bio-Rad CFX96 machine and analyzed using Bio-Rad CFX Manager (BioRad Laboratories). For each reporter dye, the threshold line was manually adjusted to the highest fluorescence value attained from the respective negative control. To validate the qPCR parameters, DNA from isolates 49 and 57 (Table 1) was diluted in a 10-fold dilution series ranging from $100 \mathrm{ng}$ to $100 \mathrm{fg}$ and used to calculate efficiencies (Broeders et al. 2014; Bustin et al. 2009). To determine the specificity of the qPCR assay to detect the E198A mutation in C. beticola, $10 \mathrm{ng}$ of template DNA from C. beticola isolates 22, 44, and 64 (Table 1), A. alternata, $P$. betae, and noninoculated sugar beet leaf samples were utilized.

Development of probe-based qPCR methodology for DMI resistance detection in $\boldsymbol{C}$. beticola. We have recently utilized a genome-wide association study to identify mutations associated with DMI resistance in C. beticola (R. Spanner and M. D Bolton, unpublished). Using this strategy, we identified a leucine to phenylalanine exchange at position 144 (L144F) encoded in the C. beticola Cyp51 gene. Additionally, we identified a synonymous mutation at amino acid position 170 (E170), also highly associated with DMI resistance in the C. beticola Cyp51 gene. To develop a molecular assay, we developed dual-labeled probes specific to each mutation for the detection and differentiation of each genotype. For optimization of each primer/probe combination, pairwise comparisons of primer (100, 200 , and $300 \mathrm{nM})$, probe $(100,200$, and $300 \mathrm{~nm}), \mathrm{MgCl}_{2}$ concentrations $(3.0,4.0$, and $5.0 \mathrm{mM})$, and annealing temperature $\left(55\right.$ to $\left.65^{\circ} \mathrm{C}\right)$ were assessed. The optimized procedure used for screening isolates in this study utilized the GoTaq G2 Flexi DNA Polymerase kit in a $25-\mu$ l volume containing $1 \times$ buffer, $4.0 \mathrm{mM} \mathrm{MgCl} 2,0.3 \mathrm{mM}$ dNTPs, $200 \mathrm{nM}$ each primer (primers 2288 and 2250) Table 2), $200 \mathrm{nM}$ each probe, $1.25 \mathrm{U}$ of GoTaq, and $10 \mathrm{ng}$ of genomic DNA. Probes 2373, 2284 , and 2445 (Table 2) were designed to differentiate the wild type (WT) from the C-type and T-type L144F mutations, respectively. Likewise, probes 2257 and 2258 (Table 2) differentiated WT from E170 mutants. qPCR conditions were $95^{\circ} \mathrm{C}$ for $10 \mathrm{~min}$, followed by 40 cycles of $95^{\circ} \mathrm{C}$ for $15 \mathrm{~s}$ and $63^{\circ} \mathrm{C}$ for $45 \mathrm{~s}$. All qPCR assays were performed in a Bio-Rad CFX96 machine and analyzed using Bio-Rad CFX Manager (Bio-Rad Laboratories). For each reporter dye, the threshold line was manually adjusted to the highest fluorescence value attained from the respective negative control. To validate the qPCR parameters, DNA from isolates 24,44 , and 81 (Table 1) was diluted in a 10-fold dilution series ranging from $100 \mathrm{ng}$ to 100 fg and used to calculate efficiencies (Broeders et al. 2014; Bustin et al. 2009). To determine the specificity of the qPCR assay to detect the $\mathrm{L} 144 \mathrm{~F}$ and E170 mutations in C. beticola, $10 \mathrm{ng}$ of template DNA from $C$. beticola isolates 22, 44, and 64 (Table 1), A. alternata, $P$. betae, and noninoculated sugar beet leaf samples were utilized.

Plant growth, inoculations, DNA isolation, and experimental design. Sugar beet seed of the CLS-susceptible cultivar Crystal 093RR (American Crystal Sugar) were planted into $15-\mathrm{cm}$ diameter plastic pots filled with Sunshine potting soil mix number 1 (Sun Gro Horticulture). Three seeds were planted per pot. Pots were placed in a greenhouse with an average daytime temperature of $24^{\circ} \mathrm{C}$, an average nighttime temperature of $16^{\circ} \mathrm{C}$, and a 16 -h photoperiod. Plants were watered as needed and each pot was thinned to one plant after seedling establishment. At 5 weeks after planting, which represents the stage at which at least six fully expanded leaves were present, plants were used in experiments described below.

C. beticola spores were produced following the methodology described by Secor and Rivera (2012) from each isolate (Table 1). Plants were inoculated with a suspension of $10^{5}$ spores $\mathrm{ml}^{-1}$ using a hand-held Preval sprayer (Preval) on the underside of the leaf and marked with a marker on the petiole of inoculated leaves. After inoculation, plants were incubated in a humidity chamber for 7 days at 95 to $99 \%$ relative humidity with a 13 -h photoperiod at $25^{\circ} \mathrm{C}$. After

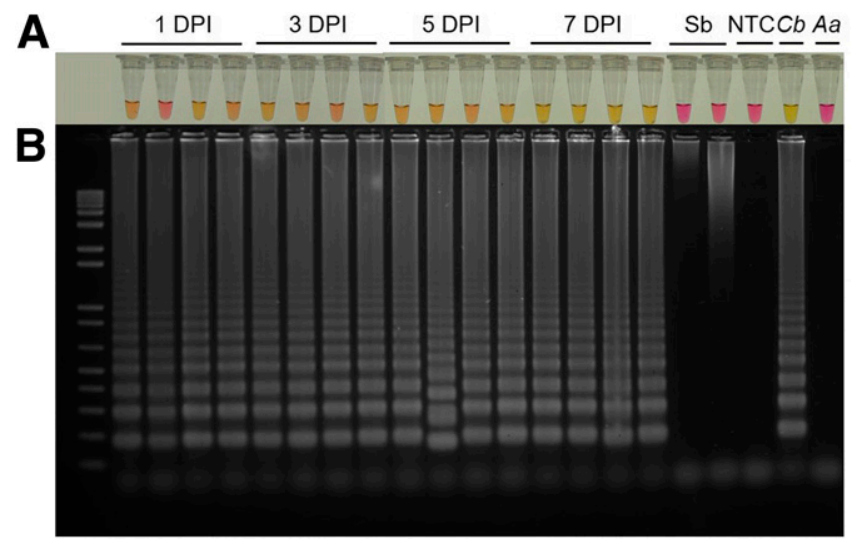

Fig. 1. Detection of Cercospora beticola in sugar beet samples at different time points. Five-week-old sugar beet plants were inoculated with $C$. beticola. Leaf discs $(5 \mathrm{~mm})$ were collected at different time points (1, 3, 5, and 7 days postinoculation [DPI]). For each time point, three leaf discs were collected from each of two leaves from each of two plants for, in total, 12 leaf discs per time point. The loop-mediated isothermal amplification (LAMP) primers specific to C. beticola (Cb-LAMP) assay was conducted on in planta DNA samples, noninoculated sugar beet control (Sb), nontemplate control (NTC), $10 \mathrm{ng}$ of $C$. beticola $(C b)$, as positive control, and Alternaria alternata $(A a)$ as negative control. Reactions were visually inspected to observe positive reaction represented by $\mathbf{A}$, the change in color from pink to yellow and $\mathbf{B}$, the presence of ladder-like bands in $2 \%$ agarose gel electrophoresis. 
this time period, plants were grown in the greenhouse under conditions described above.

Experiment I. This experiment was designed to assess whether $C$. beticola could be detected in asymptomatic leaf tissue. Isolate 57 (Table 1) was used for this experiment. Plants were inoculated as described above. Noninoculated plants served as negative controls. Samples were harvested at five time points $(1,3,5,7$, and 9 days postinoculation [DPI]). For each time point, two leaves from each of two infected plants were used for $C$. beticola detection. Additionally, two leaves from each of two noninoculated plants were used for negative controls. Leaf discs were collected using a 5-mm paper punch from each leaf and stored at $-80^{\circ} \mathrm{C}$ until use for analyses. Efficiency of the Cb-LAMP assay was assessed by calculating the percentage of positive reactions per time point (i.e., total number of positive reactions divided by the total number of reactions per time point and multiplied by 100). There were 12 samples per time point - three leaf discs per inoculated leaf (collected each from top, middle, and bottom portions) $\times$ two leaves per plant $\times$ two plants per time point—and each sample was tested five times giving, in total, 60 reactions per time point.

Experiment II. This experiment was designed to assess whether mutations associated with fungicide resistance in $C$. beticola could be identified in infected leaf tissues using LAMP- or probe-based detection strategies. One 5-week-old sugar beet plant was inoculated with each of 20 different isolates of $C$. beticola (Table 1). Inocula preparations and inoculations were done as explained in experiment I, except a suspension of $10^{4}$ spores $\mathrm{ml}^{-1}$ was used. Upon the appearance of leaf spots (12 DPI), 105 -mm leaf discs containing individual leaf spots were harvested for each isolate and stored at $-80^{\circ} \mathrm{C}$ until used for analysis.
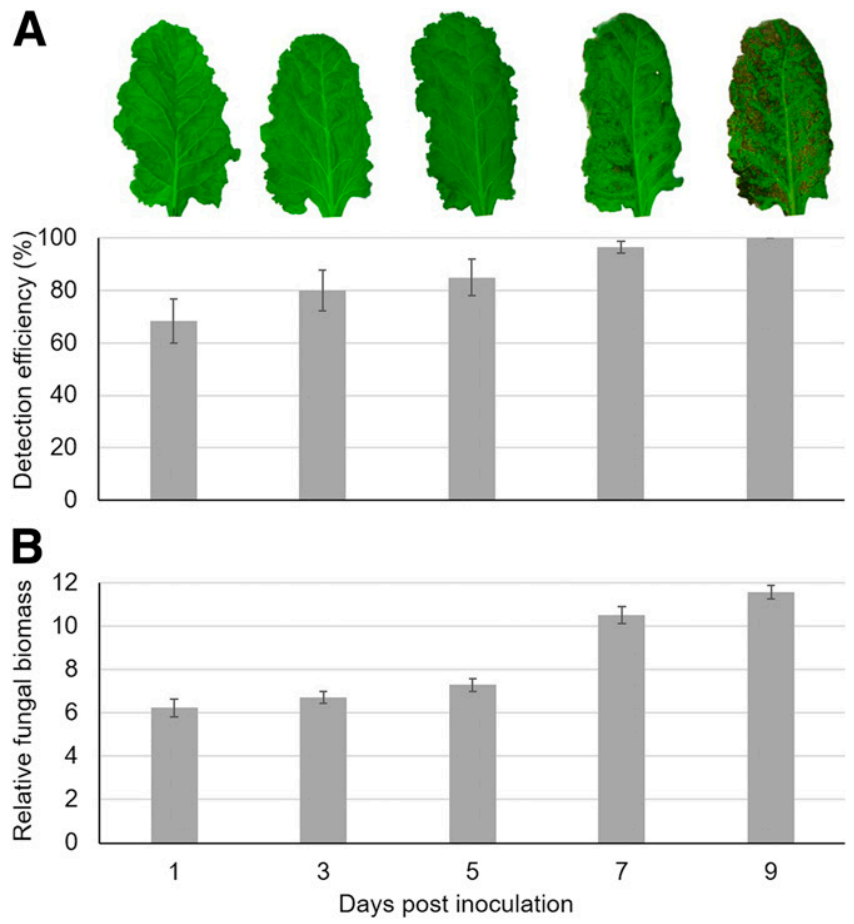

Fig. 2. Detection efficiency of the loop-mediated isothermal amplification (LAMP) primers specific to Cercospora beticola (Cb-LAMP) assay. Five-week-old sugar beet plants were inoculated with $C$. beticola. Leaf discs $(5 \mathrm{~mm})$ were collected at different time points $(1,3,5,7$, and 9 days postinoculation). For each time point, three leaf discs were collected from each of two leaves from each of two plants for, in total, 12 leaf discs per time point. Leaves on the top of the figure represent the different stage of disease progression at each time point referred to in the graphs. A, Detection efficiency was calculated by dividing the total number of positive reactions by total number of reactions per time point and multiplied by 100 . B, Natural log of relative fungal biomass of $C$. beticola to the sugar beet at different time points using quantitative PCR targeting rDNA of $C$. beticola and sugar beet. Error bars in the graphs represent the standard error of the mean of 12 replications for each time point.
For both experiments I and II, genomic DNA was extracted from each leaf disc using a 30-s nucleic acid purification method as explained by Zou et al. (2017), with few modifications. A 5-mm leaf disc was ground using a plastic pestle in a $1.5-\mathrm{ml}$ centrifuge tube containing $300 \mu \mathrm{l}$ of lysis buffer ( $20 \mathrm{mM}$ Tris [pH 8.0], $25 \mathrm{mM} \mathrm{NaCl}$, $2.5 \mathrm{mM}$ EDTA, and $0.05 \%$ sodium dodecyl sulfate). Samples were diluted 10-fold by adding $20 \mu \mathrm{l}$ of the lysed samples to $180 \mu \mathrm{l}$ of AE buffer (10 mM Tris-Cl and 0.5 mM EDTA, pH 9.0). The diluted samples were later used as a template for the LAMP assay and qPCR assays.

\section{Results}

Detection of $C$. beticola in sugar beet leaves using LAMP. A LAMP assay was developed to specifically detect $C$. beticola in sugar beet leaves by testing a variety of primers and optimization of incubation parameters (Supplementary Fig. S2). Ultimately, the primer set Cb-LAMP-2 designed on a $C$. beticola rDNA sequence using a $65^{\circ} \mathrm{C}$ incubation for 90 min was identified as the most ideal for detecting C. beticola. rDNA was chosen as a potential target for LAMP-based detection, in part because the sequence is multicopy, therefore providing additional templates from each $C$. beticola cell. Additionally, a query to over 250 C. beticola genome sequences (R. Spanner and M. D. Bolton, unpublished) did not identify sequence variation in the region where primers were designed. The Cb-LAMP assay was able to detect $C$. beticola DNA down to $100 \mathrm{fg}$ (Supplementary Fig. S3). The Cb-LAMP assay detected C. beticola in asymptomatic leaf tissue as early as 1 DPI (Fig. 1A). Characteristic "laddering" confirmed amplification (Fig. 1B). However, the efficiency of detecting $C$. beticola at early time points was lower compared
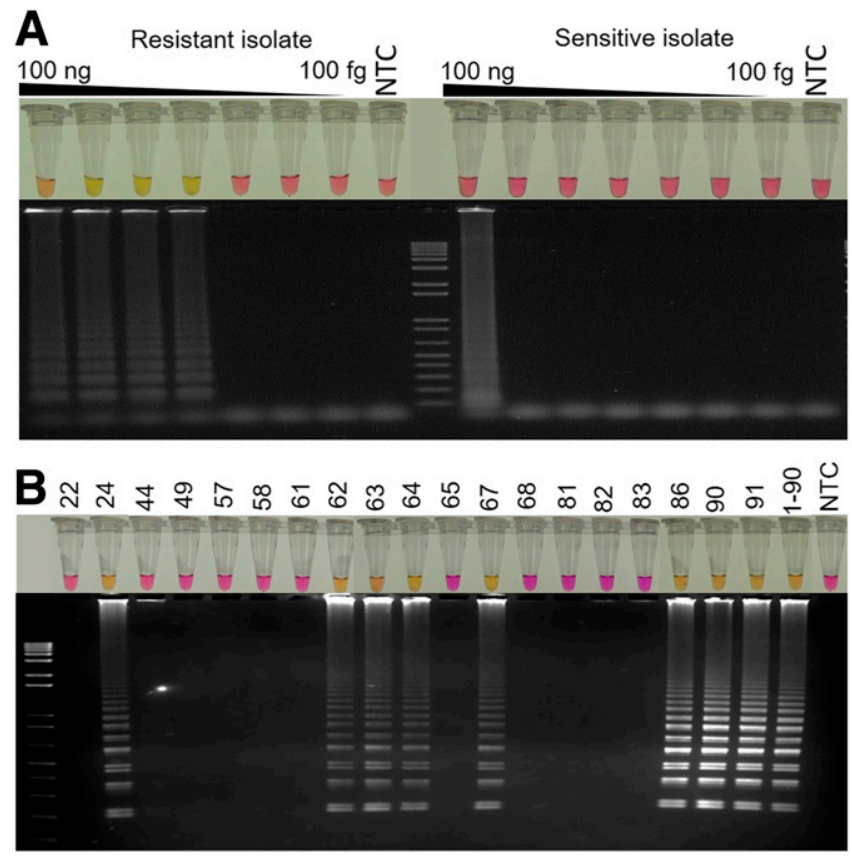

Fig. 3. Detection limit and accuracy of quinone outside inhibitor (Qol) loop-mediated isothermal amplification (LAMP) primer sets. A, Detection limit of Qol-LAMP assay was carried out by using a panel of 10 -fold dilution series ranging from $100 \mathrm{ng}$ to $100 \mathrm{fg}$ of genomic DNA (indicated by the solid black triangle on the top of reaction) from one resistant and one sensitive isolate of Cercospora beticola and a nontemplate control (NTC). Reactions were visualized for a change in color from pink to yellow in the positive reactions as well as the presence of ladder-like bands in $2 \%$ agarose gel. B, A panel of 20 different isolates of Cercospora beticola was tested for the accuracy of Qol-LAMP assay. Reactions were prepared using $10 \mathrm{ng}$ of DNA, incubated at $65^{\circ} \mathrm{C}$ for 45 min and visually inspected for the change in color from pink to yellow for positive reaction and ladder-like bands in $2 \%$ agarose gel. Numbers on the top of reaction tubes indicate different $C$. beticola isolates used in the reaction. The Qol-LAMP assay was able to amplify specifically resistant strains shown by the change of color from pink to orange and presence of ladderlike bands in $2 \%$ agarose gel. 
with later time points (Fig. 2A). Additionally, we developed a qPCR assay (Supplementary Fig. S5A) to detect $C$. beticola in asymptomatic plants. Using this assay, fungal biomass was shown to increase in sugar beet leaves after inoculation, which supported LAMP results (Fig. 2B).

Detection of QoI resistance using LAMP. A lamp assay to detect the G143A mutation in Cytb associated with QoI resistance in $C$. beticola was developed by testing FIP primers, each containing a different mismatch base at the penultimate position of the primer (Supplementary Fig. S1), and optimization of incubation parameters (Supplementary Fig. S4). The primer sets (QoI-LAMP mut-1) with [C] mismatch nucleotide and incubation at $65^{\circ} \mathrm{C}$ for $45 \mathrm{~min}$ were identified as ideal parameters for detecting the G143A mutation. The QoI-LAMP assay allowed the detection of the G143A mutation in samples with DNA concentration as low as 100 pg (Fig. 3A). Using our panel of $20 \mathrm{C}$. beticola isolates, the QoI-LAMP assay detected QoI-resistant C. beticola isolates with $100 \%$ accuracy (Fig. 3B). Results were confirmed with the probe-based qPCR assay to detect the G143A mutation previously developed by Bolton et al. (2013b) (data not shown). Likewise, the QoI-LAMP assay was used on sugar beet leaves inoculated with the same panel of 20 C. beticola isolates. A rapid DNA extraction coupled with the QoI-LAMP assay was able to detect the G143A mutation in C. beticola in infected leaf tissue with $100 \%$ accuracy (Fig. 4).

Detection of benzimidazole resistance in $C$. beticola using probe-based qPCR. A probe-based qPCR assay was developed to detect the E198A mutation in $\beta$-tubulin associated with resistance to benzimidazole in $C$. beticola. Utilizing primers and dual-labeled probes under optimized parameters exhibiting the highest efficiency and specificity (Table 2; Supplementary Fig. S5B), the assay was able to identify resistant and sensitive isolates with $100 \%$ accuracy in vitro (Fig. 5A; Supplementary Fig. S6A) and in planta (Fig. 5B) compared with the derived $\beta$-tubulin gene sequence from each isolate. Using our panel of $20 \mathrm{C}$. beticola isolates, quantitation cycle (Cq) values from sensitive isolates were achieved only with the qPCR probe specific to the sensitive allele (Fig. 5). Likewise, Cq values from resistant strains were only achieved when using the probe specific to the resistant allele (Fig. 5).

Detection of DMI resistance in $C$. beticola using probebased qPCR. We have recently identified two mutations associated

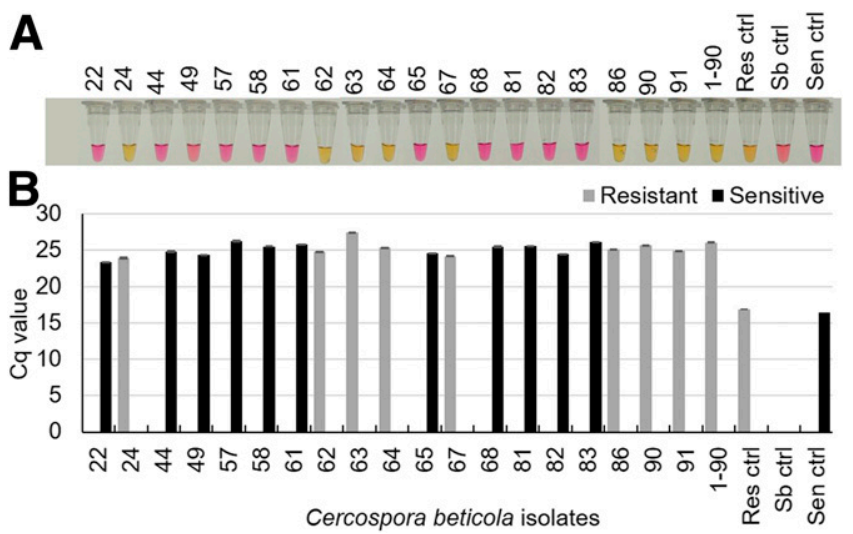

Fig. 4. Detection of the G143A mutation in cytochrome $b$ associated with $A$, quinone outside inhibitor (Qol) resistance in Cercospora beticola in sugar beet leaves using loop-mediated isothermal amplification (LAMP) and B, probe-based quantitative PCR. Five-week-old sugar beet plants were inoculated with the panel of 20 different isolates. Samples were harvested at 12 days postinoculation for analysis. Three leaf discs were analyzed for each isolate. The Qol-LAMP assay was visualized for a change in color from pink to yellow, indicative of Qol-resistant strains. One representative LAMP reaction of the three analyzed leaf discs is shown. Numbers on the top of reaction tubes indicate isolates used for inoculating sugar beet plants. The $\mathrm{Y}$-axis represents the quantitation cycle $(\mathrm{Cq})$ values and $\mathrm{X}$-axis represents the isolates and the controls used in the assay. Black bars in the graph are the $\mathrm{Cq}$ values of resistant isolates with resistant-specific probe and gray bars represent the $\mathrm{Cq}$ values of sensitive isolates with sensitive-specific probe. Error bars in this graph represent the standard error of the mean of three replications. with DMI resistance in C. beticola (R. Spanner and M. D. Bolton, unpublished). Based on these results, several primers and probes were tested to detect Cyp51 mutations encoding L144F as well as a silent mutation at amino acid E170. Because two codons were identified that encode for L144F, two probes were developed specific to these alleles. Specifically, primers 2288 and 2250 were used to amplify the region in Cyp 51 containing the mutations where probes 2373 (WT), 2284 (L144F C-type), and 2445 (L144F T-type) were used to differentiate the L144F mutation, and probes 2257 (WT) and 2258 (E170) were used to distinguish the silent mutation at E170 (Table 2; Supplementary Figs. S5C and D and S6B and C). The optimized parameters were used on 20 different isolates of $C$. beticola (Table 1) to detect these mutations, which exhibited $100 \%$ accuracy. Using the developed probes and qPCR conditions, $\mathrm{Cq}$ values were only obtained when probes matched Cyp51 gene sequences (Figs. 6 and 7).

\section{Discussion}

Sugar beet growers in North Dakota and Minnesota have suffered recurrent problems in CLS management largely due to fungicide failure. For instance, CLS management in the late 1970s mainly depended on three benzimidazole fungicides but efficacy of these fungicides reduced rapidly in $C$. beticola populations of North Dakota and Minnesota, causing an epidemic in 1981 (Smith and Cattanach 1982). Subsequently, triphenyltin hydroxide became popular among growers for CLS management but the chemistry was no longer effective due to development of fungicide resistance in 1994 (Bugbee 1995, 1996) and later led to a major epidemic in 1998 (Secor et al. 2010). Consequently, the Environmental Protection Agency granted an emergency exemption for the use of tetraconazole for sugar beet growers but resistance to this class has also been growing (Bolton et al. 2012b). QoI fungicides were also used extensively for CLS management, which contributed to resistance in many $C$. beticola populations worldwide (Birla et al. 2012; Bolton et al. 2013a,c). From 1999 to 2015 , the disease was generally well managed using fungicides of different chemistries in rotation. However, repeated and widespread use of these fungicides together with conducive weather for CLS disease development caused CLS epidemics in 2016 to 2018 in many sugar-beet-growing regions in the United States.

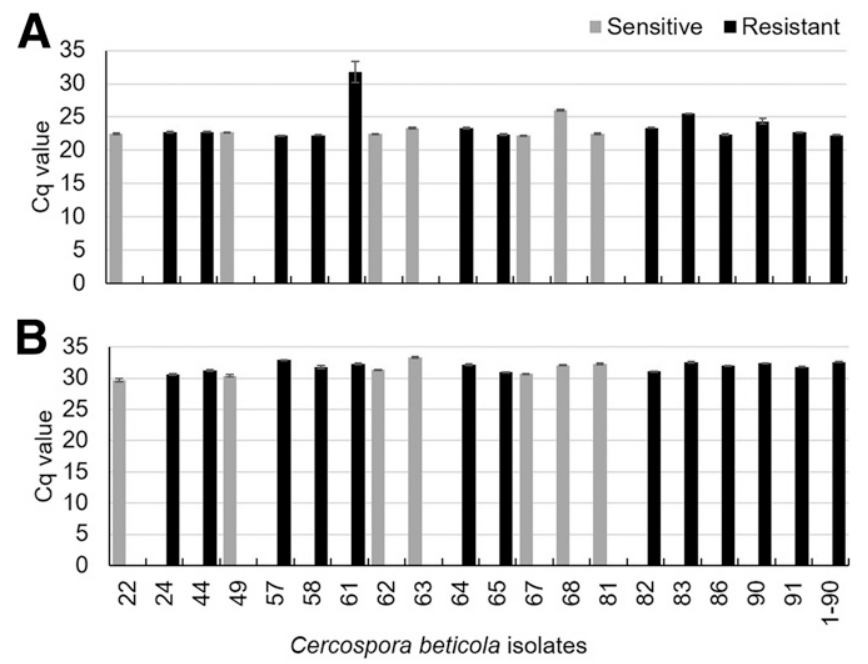

Fig. 5. Detection of the E198A mutation in $\beta$-tubulin of Cercospora beticola associated with benzimidazole resistance using probe-based quantitative PCR. A, A panel of 20 C. beticola was tested in the assay where $10 \mathrm{ng}$ of genomic DNA from in vitro samples was used. B, Five-week-old sugar beet plants were inoculated with the panel of 20 different isolates. Samples were harvested at 12 days postinoculation for analysis. Three leaf discs were analyzed for each isolate. The $\mathrm{Y}$-axis in both figures represents the quantitation cycle $(\mathrm{Cq})$ values and $X$-axis represents the isolates. Black bars in the graph are the $\mathrm{Cq}$ values of benzimidazole-resistant isolates and gray bars represent the $\mathrm{Cq}$ values of benzimidazole-sensitive isolates. Error bars in both graphs represent the standard error of the mean of three replications. 
For effective management of CLS, the timing of fungicide application is critical (Karadimos and Karaoglanidis 2006; Secor et al. 2010). However, efficacy will diminish over time if applications are made too early. Consequently, confirmation of the pathogen in leaf tissue early in the infection process can guide fungicide application decisions. In this study, we developed a LAMP assay to accurately detect $C$. beticola down to $100 \mathrm{fg}$. The aim of the in planta LAMP assay was not only to accurately detect $C$. beticola in asymptomatic leaves but also to find the earliest time point that can provide a correct diagnosis. To achieve this objective, a time series experiment was carried out using five different time points. The $\mathrm{Cb}$ LAMP assay could detect $C$. beticola as early as 1 DPI. However, detection efficiency at the early time points was relatively lower, presumably because fungal tissue was not ubiquitously present in leaf tissue at that early stage. However, the detection efficiency was almost $100 \%$ at the final time point (9 DPI). In order to confirm the presence of $C$. beticola in asymptomatic plants early in the infection process, samples should be collected and assessed from several leaves in a field.

Once $C$. beticola has been confirmed in a field, growers must decide which fungicide to apply for disease management. Clearly, this decision must consider fungicide resistance occurring in the field. To that end, our fungicide resistance detection toolbox contains three new assays to quickly identify mutations associated with fungicides used for CLS. To detect QoI resistance, a LAMP assay was developed. Although resistance to QoIs has been widespread in some $C$. beticola populations, several sugar-beet-growing regions utilize this chemistry for disease management. Therefore, a LAMP assay was developed for rapid detection of the mutation and early decision making regarding the type of fungicide to apply. The optimized QoI-LAMP assay established in our study was able to detect the G143A mutation in C. beticola in vitro and in planta with $100 \%$ accuracy.

This study was also focused on developing probe-based qPCR to detect different mutations associated with resistance to chemistries commonly used for CLS disease management. Until this study, the only probe-based qPCR assay developed was designed to identify the G143A mutation involved with QoI resistance in $C$. beticola (Bolton et al. 2013a). In the current study, we utilized mutations associated with benzimidazole resistance (Bolton et al. 2013b; Trkulja et al. 2013) and novel mutations associated with DMI resistance (R. Spanner and M. D. Bolton, unpublished) to develop primers and probes specific to the fungicide-sensitive WT and fungicideresistant mutants and optimize the probe-based qPCR assays.

We also attempted to identify the E198A mutation associated with benzimidazole resistance using LAMP techniques. However, reproducibility was very low due to apparently random and frequent falsepositive issues that occurred with all attempted primer designs (data not shown). There are no conclusive explanations behind such observations. However, the probe-based qPCR was developed to detect the E198A mutation in C. beticola in vitro and in planta with $100 \%$ accuracy.

DMIs are curative fungicides, making them popular with growers. However, resistance in some $C$. beticola populations has become a serious problem (Bolton et al. 2012a, 2016). Recently, we have identified two mutations in Cyp51 (L144F and a silent mutation at amino acid E170) associated with DMI resistance (R. Spanner and M. D. Bolton, unpublished ). We were able to develop a highly sensitive probe-based qPCR to accurately detect these mutations in $C$. beticola in vitro and in planta.

In conclusion, this study demonstrated that LAMP can be used for the accurate and efficient detection of $C$. beticola in infected sugar beet leaves. The LAMP assay is a simple, sensitive, and reliable technique that can amplify DNA at a single temperature. Therefore, LAMP does not require specialized equipment such as a PCR machine. In fact, the heat block used in this study runs on $12 \mathrm{~V}$, rendering the possibility that CLS diagnosis could be carried out using standard auxiliary power from a vehicle in the field. Moreover, the DNA isolation technique used in this study is also simple and does not require the use of specialized equipment. Taken together, the LAMP-based detection methodology developed here has great potential for specific identification of $C$. beticola in the field, which will help the grower make timely fungicide application decisions.

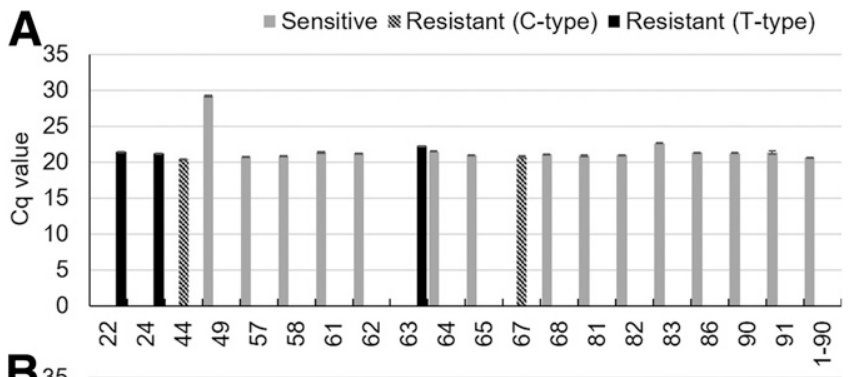

$\mathbf{B}_{35}$

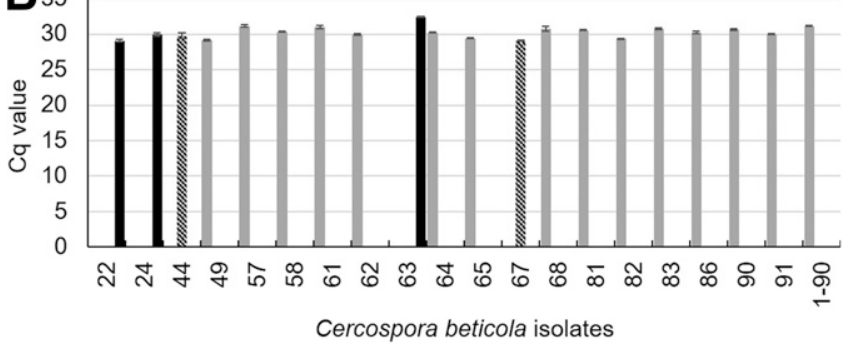

Fig. 6. Detection of the L144F mutation in Cyp51 of Cercospora beticola associated with sterol demethylation inhibitor (DMI) resistance using probe-based quantitative PCR. A, A panel of $20 \mathrm{C}$. beticola was tested in the assay using $10 \mathrm{ng}$ of genomic DNA from in vitro samples. B, Five-week-old sugar beet plants were inoculated with the panel of 20 different isolates. Samples were harvested at 12 days postinoculation for analysis. Three leaf discs were analyzed for each isolate. The $Y$ axis in both figures represents the quantitation cycle $(\mathrm{Cq})$ values and $\mathrm{X}$-axis represents the isolates and the controls used in the assays. Black bars in the graph are the $\mathrm{Cq}$ values of DMl-resistant isolates (L144F, T-type), hatched gray bars are DMI-resistant isolates (L144F, C-type), and gray bars represent the Cq values of sensitive isolates. Error bars in both graphs represent the standard error of the mean of three replications.

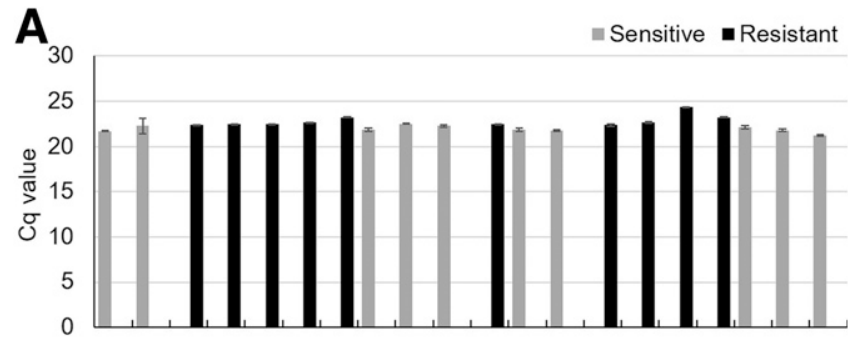

B

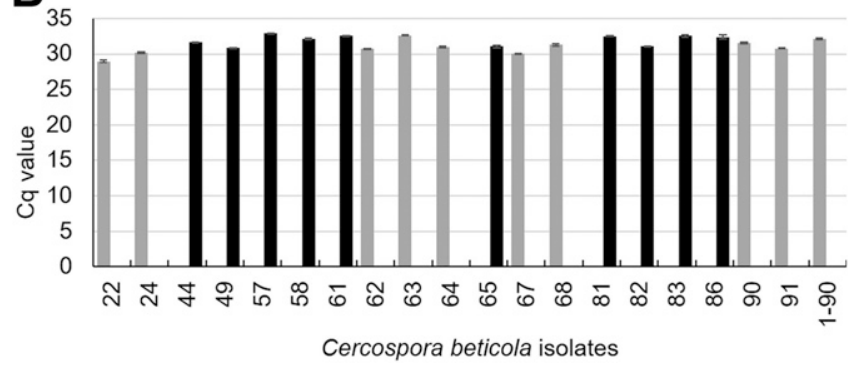

Fig. 7. Detection of the E170 mutation in Cyp51 of Cercospora beticola associated with sterol demethylation inhibitor (DMI) resistance using probe-based quantitative PCR. A, A panel of $20 \mathrm{C}$. beticola was tested in the assay using $10 \mathrm{ng}$ of genomic DNA from in vitro samples. B, Five-week-old sugar beet plants were inoculated with the panel of 20 different isolates. Samples were harvested at 12 days postinoculation for analysis. Three leaf discs were analyzed for each isolate. The $Y$-axis in both figures represents the quantitation cycle $(\mathrm{Cq})$ values and $X$-axis represents the isolates and the controls used in the assays. Black bars in the graph are the $\mathrm{Cq}$ values of DMI-resistant isolates and gray bars represent the $\mathrm{Cq}$ values of sensitive isolates. Error bars in both graphs represent the standard error of the mean of three replications. 


\section{Acknowledgments}

We thank A. Dessert for helping in planting and inoculating sugar beet plants and V. Rivera-Varas (North Dakota State University) for excellent technical assistance.

\section{Literature Cited}

Birla, K., Rivera-Varas, V., Secor, G. A., Khan, M. F., and Bolton, M. D. 2012. Characterization of cytochrome $\mathrm{b}$ from European field isolates of Cercospora beticola with quinone outside inhibitor resistance. Eur. J. Plant Pathol. 134: 475-488.

Bolton, M. D., Birla, K., Rivera-Varas, V., Rudolph, K. D., and Secor, G. A. 2012a. Characterization of CbCyp51 from field isolates of Cercospora beticola. Phytopathology 102:298-305.

Bolton, M. D., Ebert, M. K., Faino, L., Rivera-Varas, V., de Jonge, R., Van de Peer, Y., Thomma, B. P., and Secor, G. A. 2016. RNA-sequencing of Cercospora beticola DMI-sensitive and-resistant isolates after treatment with tetraconazole identifies common and contrasting pathway induction. Fungal Genet. Biol. 92:1-13.

Bolton, M. D., Rivera, V., and Secor, G. 2013a. Identification of the G143A mutation associated with QoI resistance in Cercospora beticola field isolates from Michigan, United States. Pest Manage. Sci. 69:35-39.

Bolton, M. D., Rivera-Varas, V., Khan, M. F., and Secor, G. 2013b. PCR-based detection of benzimidazole resistance in Cercospora beticola. Sugarbeet Research and Education Board Research Reports. https://www.sbreb.org/ research/

Bolton, M. D., Rivera-Varas, V., Secor, G. A., Cattanach, A. W., and Metzger, M. S. 2013c. Identification of the G143A mutation in cytochrome b associated with QoI resistance in Cercospora beticola isolates from the Red River Valley. Plant Health Prog. 14. doi:10.1094/PHP-2013-0812-02-RS

Bolton, M. D., Secor, G. A., Rivera, V., Weiland, J. J., Rudolph, K., Birla, K., Rengifo, J., and Campbell, L. G. 2012b. Evaluation of the potential for sexual reproduction in field populations of Cercospora beticola from USA. Fungal Biol. 116:511-521.

Broeders, S., Huber, I., Grohmann, L., Berben, G., Taverniers, I., Mazzara, M., Roosens, N., and Morisset, D. 2014. Guidelines for validation of qualitative real-time PCR methods. Trends Food Sci. Technol. 37:115-126.

Bugbee, W. 1995. Cercospora beticola tolerant to triphenyltin hydroxide. J. Sugar Beet Res. 32:167-174.

Bugbee, W. 1996. Cercospora beticola strains from sugar beet tolerant to triphenyltin hydroxide and resistant to thiophanate methyl. Plant Dis. 80:103. doi:10.1094/PD-80-0103B

Bustin, S. A., Benes, V., Garson, J. A., Hellemans, J., Huggett, J., Kubista, M., Mueller, R., Nolan, T., Pfaffl, M. W., and Shipley, G. L. 2009. The MIQE guidelines: Minimum information for publication of quantitative real-time PCR experiments. Clin. Chem. 55:611-622.

Cools, H. J., Bayon, C., Atkins, S., Lucas, J. A., and Fraaije, B. A. 2012. Overexpression of the sterol $14 \alpha$-demethylase gene (MgCYP51) in Mycosphaerella graminicola isolates confers a novel azole fungicide sensitivity phenotype. Pest Manage. Sci. 68:1034-1040.

Cools, H. J., Hawkins, N. J., and Fraaije, B. A. 2013. Constraints on the evolution of azole resistance in plant pathogenic fungi. Plant Pathol. 62:36-42.

Cools, H. J., Parker, J. E., Kelly, D. E., Lucas, J. A., Fraaije, B. A., and Kelly, S. L. 2010. Heterologous expression of mutated eburicol 14 $\alpha$-demethylase (CYP51) proteins of Mycosphaerella graminicola to assess effects on azole fungicide sensitivity and intrinsic protein function. Appl. Environ. Microbiol. 76: 2866-2872.

Davidson, R., Hanson, L., Franc, G., and Panella, L. 2006. Analysis of $\beta$-tubulin gene fragments from benzimidazole-sensitive and-tolerant Cercospora beticola. J. Phytopathol. 154:321-328.

Franc, G. D. 2010. Ecology and epidemiology of Cercospora beticola. Pages 7-19 in:Cercospora Leaf Spot of Sugar Beet and Related Species. R. T. Lartey, J. J. Weiland, L. Panella, P. W. Crous, and C. E. Windels, eds. American Phytopathological Society, St. Paul, MN, U.S.A.

Gisi, U., Sierotzki, H., Cook, A., and McCaffery, A. 2002. Mechanisms influencing the evolution of resistance to Qo inhibitor fungicides. Pest Manage. Sci. 58:859-867.

Goto, M., Honda, E., Ogura, A., Nomoto, A., and Hanaki, K.-I. 2009. Colorimetric detection of loop-mediated isothermal amplification reaction by using hydroxy naphthol blue. Biotechniques 46:167-172.

Kaiser, U., Kluth, C., and Märländer, B. 2010. Variety-specific epidemiology of Cercospora beticola Sacc. and consequences for threshold-based timing of fungicide application in sugar beet. J. Phytopathol. 158:296-306.

Karadimos, D., and Karaoglanidis, G. 2006. Comparative efficacy, selection of effective partners, and application time of strobilurin fungicides for control of Cercospora leaf spot of sugar beet. Plant Dis. 90:820-825.
Khan, J., Del Río, L., Nelson, R., and Khan, M. 2007. Improving the Cercospora leaf spot management model for sugar beet in Minnesota and North Dakota. Plant Dis. 91:1105-1108.

Kogovšek, P., Hodgetts, J., Hall, J., Prezelj, N., Nikolić, P., Mehle, N., Lenarčič, R., Rotter, A., Dickinson, M., and Boonham, N. 2015. LAMP assay and rapid sample preparation method for on-site detection of flavescence dorée phytoplasma in grapevine. Plant Pathol. 64:286-296.

Kretschmer, M., Leroch, M., Mosbach, A., Walker, A.-S., Fillinger, S., Mernke, D., Schoonbeek, H.-J., Pradier, J.-M., Leroux, P., and De Waard, M. A 2009. Fungicide-driven evolution and molecular basis of multidrug resistance in field populations of the grey mould fungus Botrytis cinerea. PLoS Pathogens 5:e1000696.

Ma, B., and Tredway, L. P. 2013. Induced overexpression of cytochrome P450 sterol $14 \alpha$-demethylase gene (CYP51) correlates with sensitivity to demethylation inhibitors (DMIs) in Sclerotinia homoeocarpa. Pest Manage. Sci. 69:1369-1378.

Miller, J., Rekoske, M., and Quinn, A. 1994. Genetic resistance, fungicide protection and variety approval policies for controlling yield losses from Cercospora leaf spot infections. J. Sugar Beet Res. 31:7-12.

Nagamine, K., Hase, T., and Notomi, T. 2002. Accelerated reaction by loopmediated isothermal amplification using loop primers. Mol. Cell. Probes 16: 223-229.

Obuya, J. O., Ananga, A., and Franc, G. D. 2015. Silent mutation: Characterization of its potential as a mechanism for sterol $14 \alpha$-demethylase resistance in Cercospora beticola field isolates from the United States. J. Plant Pathol. Microbiol. 6.

Omrane, S., Audéon, C., Ignace, A., Duplaix, C., Aouini, L., Kema, G., Walker, A.-S., and Fillinger, S. 2017. Plasticity of the MFS1 promoter leads to multidrug resistance in the wheat pathogen Zymoseptoria tritici. MSphere 2: e00393-17.

Ordóñez, N., Salacinas, M., Mendes, O., Seidl, M., Meijer, H., Schoen, C., and Kema, G. 2019. A loop-mediated isothermal amplification (LAMP) assay based on unique markers derived from genotyping by sequencing data for rapid in planta diagnosis of Panama disease caused by tropical race 4 in banana. Plant Pathol. 68:1682-1693.

Pethybridge, S. J., Sharma, S., Hansen, Z., Vaghefi, N., Hanson, L. E., and Kikkert, J. R. Improving fungicide-based management of Cercospora leaf spot in table beet in New York, USA. Can. J. Plant Pathol. In press. https://doi.org/ 10.1080/07060661.2019.1690048

Pfaffl, M. W. 2001. A new mathematical model for relative quantification in realtime RT-PCR. Nucleic Acids Res. 29:e45.

Pitblado, R. 2002. BeetCast. www.michiganbeets.com

Secor, G. A., and Rivera, V. V. 2012. Fungicide resistance assays for fungal plant pathogens. Pages 385-392 in: Plant Fungal Pathogens. M. Bolton and B. Thomma, eds. Methods in Molecular Biology (Methods and Protocols), vol. 835. Springer. https://doi.org/10.1007/978-1-61779-501-5_23 in:

Secor, G. A., Rivera, V. V., Khan, M., and Gudmestad, N. C. 2010. Monitoring fungicide sensitivity of Cercospora beticola of sugar beet for disease management decisions. Plant Dis. 94:1272-1282.

Smith, L., and Cattanach, A. 1982. Response of sugarbeet varieties to various fungicides and fungicide combinations. Sugarbeet Res. Ext. Rep. 13: 184-194.

Steddom, K., Bredehoeft, M., Khan, M., and Rush, C. 2005. Comparison of visual and multispectral radiometric disease evaluations of Cercospora leaf spot of sugar beet. Plant Dis. 89:153-158.

Trkulja, N., Ivanović, Ž., Pfaf-Dolovac, E., Dolovac, N., Mitrović, M., Toševski, I., and Jović, J. 2013. Characterisation of benzimidazole resistance of Cercospora beticola in Serbia using PCR-based detection of resistanceassociated mutations of the $\beta$-tubulin gene. Eur. J. Plant Pathol. 135:889-902.

Trkulja, N. R., Milosavljević, A. G., Mitrović, M. S., Jović, J. B., Toševski, I. T., Khan, M. F., and Secor, G. A. 2017. Molecular and experimental evidence of multi-resistance of Cercospora beticola field populations to MBC, DMI and QoI fungicides. Eur. J. Plant Pathol. 149:895-910.

Vielba-Fernández, A., de Vicente, A., Pérez-García, A., and Fernández-Ortuño, D. 2019. Monitoring methyl benzimidazole carbamate-resistant isolates of the cucurbit powdery mildew pathogen, Podosphaera xanthii, using loopmediated isothermal amplification. Plant Dis. 103:1515-1524.

Windels, C. E., Lamey, H. A., Hilde, D., Widner, J., and Knudsen, T. 1998. A Cercospora leaf spot model for sugar beet: In practice by an industry. Plant Dis. 82:716-726.

Zou, Y., Mason, M. G., Wang, Y., Wee, E., Turni, C., Blackall, P. J., Trau, M., and Botella, J. R. 2017. Nucleic acid purification from plants, animals and microbes in under 30 seconds. PLoS Biol. 15:e2003916.

Zwiers, L.-H., Stergiopoulos, I., Van Nistelrooy, J. G., and De Waard, M. A. 2002. ABC transporters and azole susceptibility in laboratory strains of the wheat pathogen Mycosphaerella graminicola. Antimicrob. Agents Chemother. 46: 3900-3906. 